\section{Influenza vaccination of health care workers}

To support his argument in favour of compulsory influenza vaccination of health care workers, Flegel ${ }^{1}$ cites a Cochrane review ${ }^{2}$ as evidence of the vaccine's benefit.

However the following points should be noted:

- Flegel fails to convey to readers the Cochrane review's warning: "All findings must be interpreted with caution given the presence of selection bias."

- The author has cited the 2006 version of the Cochrane review, ${ }^{2}$ rather than the up-to-date version from $2010 .^{3}$

- The up-to-date Cochrane review from 2010 largely contradicts Flegel's statements, and suggests the evidence of benefit is poor. The reviewers write: "The three studies in the first publication of this review and the two new studies we identified in this update are all at high risk of bias." They summarize: "We conclude that there is no evidence that only vaccinating healthcare workers prevents laboratoryproven influenza, pneumonia, and death from pneumonia in elderly residents in long-term care facilities."

- Flegel also failed to cite another Cochrane review, which suggests alternatives to vaccination: "Implementing barriers to transmission, such as isolation, and hygienic measures (wearing masks, gloves and gowns) can be effective in containing respiratory virus epidemics or in hospital wards." ${ }^{4}$

These issues should be clarified and corrected.

Peter Doshi PhD, Elia Abi-Jaoude MSc MD, Joel Lexchin MD, Tom Jefferson MD MSc MRCGP, Roger E. Thomas MD PhD

Postdoctoral Fellow in Comparative Effectiveness Research, Divisions of General Pediatrics and General Internal Medicine (Doshi), Johns Hopkins University, School of Medicine, Baltimore, Md; Research Fellow, Department of Psychiatry (AbiJaoude), University of Toronto; Staff emergency physician, University Health
Network (Lexchin), Toronto, Ont.; Reviewer, Cochrane Collaboration (Jefferson), Rome, Italy; and Professor of Family Medicine (Thomas), University of Calgary, Calgary, Alta.

\section{References}

1. Flegel K. Health care workers must protect patients from influenza by taking the annual vaccine. CMAJ 2012;184:1873.

2. Thomas RE, Jefferson T, Demicheli V, et al. Influenza vaccination for healthcare workers who work with the elderly [review]. Cochrane Database Syst Rev 2006;(3):CD005187.

3. Thomas RE, Jefferson T, Lasserson TJ. Influenza vaccination for healthcare workers who work with the elderly. Cochrane Database Syst Rev [review] 2010;(2):CD005187.

4. Jefferson T, Del Mar C, Dooley L, et al. Physical interventions to interrupt or reduce the spread of respiratory viruses [review]. Cochrane Database Syst Rev 2010;(1):CD006207.

CMAJ 2013. DOI:10.1503/cmaj.113-2096

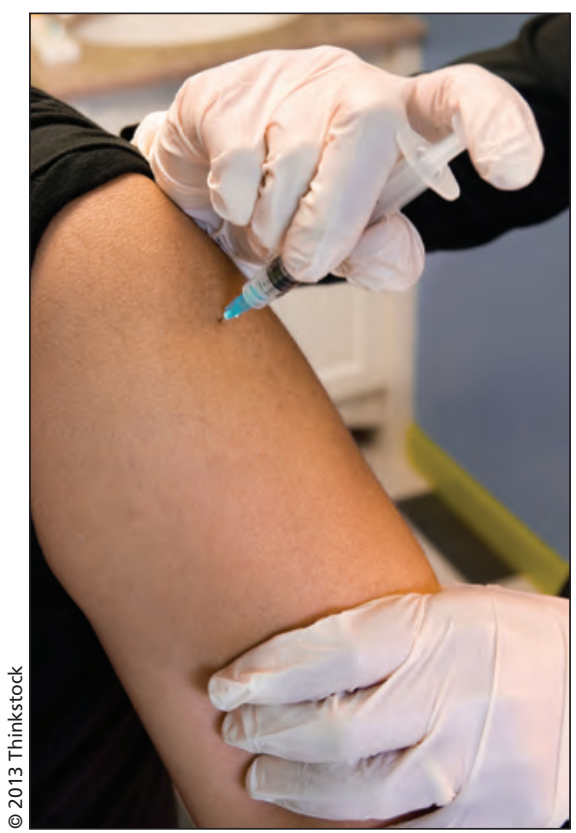

Although we strongly support increasing influenza vaccination of health care workers, we believe that guidance supporting this effort should be based on scientifically sound evidence regarding influenza vaccine effectiveness and the subsequent disease reduction in influenza workers and their patients. The editorial by Flegel contains factual errors, and the data cited to support the case for health care worker vaccination are problematic.

The vaccine efficacy rate of "about $86 \%$ when the circulating strain and vac- cine strain are well matched" is not an accurate statement, and the reference provided does not support that conclusion. ${ }^{1,2}$ We recently conducted a comprehensive meta-analysis of influenza vaccine efficacy and effectiveness and found that the trivalent inactivated vaccine provides approximately $59 \%$ protection in healthy younger adults and that match did not significantly affect how well the vaccine protected against influenza. ${ }^{3}$ Additionally, we found that the perception that current influenza vaccines provide such high levels of protection is a major barrier to developing novel-antigen, game-changing vaccines. ${ }^{4}$

Also, the 4 randomized controlled trials cited in reference 5 do not provide strong evidence to support an impact on patient mortality when increased numbers of health care workers are vaccinated. ${ }^{5}$ In fact, 2 of the studies do not support this claim, ${ }^{6,7}$ and the other 2 only weakly support it. ${ }^{8,9}$

The cost savings report cited ${ }^{10}$ (reference $^{6}$ in the editorial) uses the 2006 HICPAC statement as the source for these cost savings, but those numbers are not present in that report. ${ }^{11}$ Additionally, most cost-effectiveness numbers are based on overestimated vaccine efficacy and need to be revised. ${ }^{4}$

Last, there is no process for selecting influenza vaccine strains that reduces the risk of Guillain-Barré syndrome (GBS), as the reason influenza vaccine causes GBS is still unclear.

\section{Michael T. Osterholm PhD MPH, Nicholas S. Kelley PhD}

Center for Infectious Disease Research and Policy University of Minnesota, Minneapolis, Minn.

\section{References}

1. Flegel K. Health care workers must protect patients from influenza by taking the annual vaccine. CMAJ 2012;184:1873.

2. Thomas RE, Jefferson T, Demicheli V, et al Influenza vaccination for healthcare workers who work with the elderly [review]. Cochrane Database Syst Rev 2006;(3):CD005187.

3. Osterholm MT, Kelley NS, Sommer A, et al. Efficacy and effectiveness of influenza vaccines: a systematic review and meta- analysis. Lancet Infect Dis 2012;12:36-44.

4. Osterholm MT, Kelley NS, Manske JM, et al. The Compelling need for game-changing influenza vac cines: an analysis of the influenza vaccine enterprise and recommendations for the future. Minneapolis 